Medical Sciences and Peking Union Medical College, Beijing, China (Yjiaxin20202002@63.com)

${ }^{2}$ Shandong Cancer Hospital Affiliated to Shandong University, Shandong Academy of Medical Sciences, Jinan, China

Objective: Treatment options for platinum-resistant/refractory ovarian cancer (PROC) are few and efficacies are limited especially for primary platinum-resistant/refractory disease. PARP inhibitor (PARPi) combined with chemotherapy could improve the treatment outcome. So, we designed a phase 2 study to evaluate the efficacy and safety of niraparib combined with oral etoposide in PROC.

Methods: Eligible patients had PROC with histologically confirmed non-mucinous epithelial ovarian, fallopian tube, or primary peritoneal cancer. They must have had 1-2 prior lines of platinum-based chemotherapy, and no prior therapy with PARPi. Patients received niraparib $200 \mathrm{mg}$ or $100 \mathrm{mg}$ alternate once daily and oral etoposide $50 \mathrm{mg}$ on day 1-20 of a 30-day cycle. Oral etoposide was administered for a maximum of 6-8 cycles. Treatment was continued until disease progression, patient withdrawal or unacceptable toxic effects. The primary endpoint is progression free survival evaluated by RECIST v1.1. Results: Recruitment began on 22 May 2020. The 20 patients were enrolled to date. The mean number of prior lines of chemotherapy was 1.3 for these patients, which means almost all of them had primary platinum-resistant/refractory disease. Median follow-up period was 3.2 months (1.1-6.5). One patient achieved a partial response, 6 had stable disease (RECIST v1.1). The 15 patients had discontinued the study treatment due to disease progression or intolerable gastrointestinal toxicity. The 5 patients remained on treatment. No new safety signals were identified.

Conclusion: Niraparib combined with oral etoposide had a manageable toxicity profile and showed promising antitumor activity in PROC with 1-2 prior lines of platinum-based chemotherapy. Study recruitment is ongoing.

Trial Registration: ClinicalTrials.gov Identifier: NCT04217798

Oral (002)

Epithelial Ovarian Cancer including Borderline Tumor

https://doi.org/10.3802/jgo.2021.32.S1.002

\section{Factors predict postoperative morbidity in advanced stage ovarian cancer patients who underwent cytoreductive surgery: a systematic review and meta-analysis}

\footnotetext{
Malika Kengsakul,,", Gatske M. Nieuwenhuyzen-de Boer, ${ }^{1}$ Suwasin Udomkarnjananun, ${ }^{2}$ Stephen J. Kerr, ${ }^{3}$

Christa D. Niehot, ${ }^{4}$ Heleen J. van Beekhuizen'
}

'Department of Gynecologic Oncology, Erasmus MC Cancer Institute, University Medical Center Rotterdam, Rotterdam, The Netherlands (malika@g.swu.ac.th)

${ }^{2}$ Faculty of Medicine, King Chulalongkorn Memorial Hospital, Chulalongkorn University, Bangkok, Thailand

${ }^{3}$ Research Affairs, Faculty of Medicine, Chulalongkorn University, Bangkok, Thailand

${ }^{4}$ Medical Library, Erasmus MC Cancer Institute, University Medical Center Rotterdam, Rotterdam, The Netherlands

Objective: The cornerstone treatment for advanced stage epithelial ovarian cancer (EOC) is cytoreductive surgery followed by adjuvant chemotherapy. An independent prognostic factor for advanced stage EOC is the amount of residual disease following cytoreductive surgery. Recently, more extensive surgery has been performed in order to achieve complete cytoreduction. The aim of this study was to evaluate adjustable predictive factors for postoperative morbidity after extensive cytoreductive surgery for advanced stage EOC.

Methods: The Embase, MEDLINE, Web of Science, Cochrane Library and Google Scholar databases were searched for articles from the database inception to May 2021. Meta-analysis was conducted to determine factors associated with postoperative complication by using random-effects models.

Results: The 1,953 citations were retrieved from searching strategy. Fourteen relevant studies, involving 15,219 ovarian cancer patients, were included in the review. Severe 30day postoperative complication occurred in 2,338 (15.4\%) patients. Postoperative mortality rate was $1.93 \%$. Meta-analysis demonstrated that patient with following risk factors; age $(\mathrm{p}<0.001)$, Eastern Cooperative Oncology Group score $>0$ ( $\mathrm{p}=0.001)$, albumin level $<3.5 \mathrm{~g} / \mathrm{dL}(\mathrm{p}<0.001)$, presence of ascites on computed tomography scan $(\mathrm{p}=0.013)$, stage IV disease $(\mathrm{p}<0.001)$ and extensive surgical procedure $(\mathrm{p}<0.001)$ has a significant increase risk of developing postoperative complication. Surgical procedures including peritonectomy $(\mathrm{p}=0.012)$, splenectomy $(\mathrm{p}<0.001)$ and colon surgery $(\mathrm{p}<0.001)$ were significant predictors for postoperative complication. Conclusion: Our study demonstrated that patient's performance status and hypoalbuminemia were the only significant adjustable preoperative risk factors associated with postoperative complication. Preoperative assessment of serum albumin is essential to identify patients at risk for complication after cytoreductive surgery. A future randomized controlled trial on preoperative nutritional support and physiotherapy prehabilitation should be conducted to evaluate the impact on postoperative complication outcome. 